\title{
CIM business process and enterprise activity modelling
}

\author{
F.B. Vernadat \\ INRIA Rhône-Alpes \\ 46, avenue Félix Viallet, F-38031 Grenoble Cédex, France \\ Tel.: +3383593000,e-mail: Francois.Vernadat@inria.fr
}

\begin{abstract}
Business process and enterprise activity modelling plays a central role in enterprise modelling in the context of CIM. Business processes model enterprise behaviour while enterprise activities model enterprise functionality. In this paper, a formalism is presented to specify business processes and enterprise activities. The formalism makes use of behavioural rules derived from process algebra for structured processes and temporal logic for ill-structured processes.
\end{abstract}

Keywords

CIM, Enterprise Modelling, Business Processes, Specification

\section{INTRODUCTION}

Any enterprise can be seen as a complex dynamic system made of a set of functional entities (or agents) executing concurrent and/or cooperative processes, where each process is a flow of activities, execution of which is triggered by the occurrence of some events. Activities process manifestations or appearances of enterprise objects (either physical or informational entities).

Enterprise modelling is concerned with the representation and specification of the various aspects of enterprise operations, namely: functional aspects to describe what are the things to be done and in which order, informational aspects to describe which objects are used or processed, resource aspects to describe what or who performs things and according to which policy, and organisational aspects to describe the organisational structure and the responsibility frame within which things are being done (AMICE, 1993; CEN, 1990; Vernadat, 1995).

Within this context, enterprise behaviour and enterprise functionality need to be precisely described, specified and analysed. Enterprise behaviour concerns the order in which things are going to be executed, i.e. the flow of control. It is described in terms of business processes. Enterprise functionality concerns the things being done, i.e. the functions (either 
transformational or decision-making functions) performed by resources as set of actions. It is described in terms of enterprise activities.

\section{ENTERPRISE ACTIVITIES}

\subsection{General presentation}

The world is made of concrete and/or abstract objects. Objects are characterised by their life cycle (i.e. the succession of their states over time). Objects are visible or accessible under different forms (or embodiments) and formats (or representations). We call these object appearances or manifestations object views. One of the object views of an object is the object itself. For any object, we can distinguish two types of object views: physical object views (representing physical appearances of the object) and information object views (data representations of the object). For instance, in the case of a 50-page document, the 50 pages in paper form is a physical object view while the document information stored on disk is an information object view of the document.

This typification makes possible the separate modelling of the information flow and the material flow of an enterprise. It also makes possible to view an enterprise object in the role of a resource at certain periods of time and to view it in other roles at other periods of time.

Any time an object is created, deleted or modified in the enterprise system, this is reflected as a change in the system state. State changes are called events. Events model the fact that something has happened in the system. Requests or orders to do something are examples of events. Specific clock times can also be defined as events. Their occurrence trigger the processing of some actions (i.e. processes).

All the enterprise modelling constructs (process, activity, event, object view, functional entity) can be modelled as objects as defined in computer science, i.e. by their object identity, state (set of descriptive attributes), and behaviour (set of methods) (Rumbaugh et al. 1991).

\subsection{Enterprise activity definition and representation}

By essence, an activity $A$ is a transformational function $f$ which performs something, except the activity NIL which does nothing (neutral element in the set of activities A). By definition, an activity transforms its inputs into outputs under some conditions. In other words, it transforms an initial state into a final state under the condition $\mathrm{C}$ (a predicate called a guard). We can write:

final state $=\mathrm{f}($ initial state $)$ subject to guard $(\mathrm{f})=\mathrm{C}$

The guard can be used to specify triggering conditions and resource requirements. Let us assume that it is always possible to associate with each activity $\mathrm{A}$ of $\mathbf{A}$ a finite set ES $_{\mathbf{A}}$ of termination statuses, called ending statuses. Ending statuses are defined as 0-argument predicates. They summarise the termination status of the execution of an occurrence of the activity (such as 'successful termination', 'aborted', 'done', etc.). A generic function ES returning the ending status at the end of an activity execution can be defined as: 
$\mathrm{ES}: \mathbf{A} \rightarrow \cup \mathrm{A} \in \mathbf{A} \mathrm{ES}_{\mathrm{A}}$ such that $\mathrm{ES}(\mathrm{A}) \in \mathrm{ES}_{\mathrm{A}}$

This formal definition is not explicit enough for enterprise modelling. Different formalisms for graphical representation of activities have been proposed. First, a Generic Activity Model (GAM) has been proposed by ISO TC 184 (not a standard). It consists of a box with 10 legs as illustrated by Figure 1 (ISO, 1990). It differentiates between the information flow and the material flow and makes explicit resource inputs and outputs. It also suggests that any manufacturing activity falls under one of the generic types: transport, transform, verify, and store. However, limitations of this representation include: inputs and outputs have no precise semantics (they are just labels and no object type is defined), the model fails to describe exact triggering conditions as well as ending statuses of activities and the representation is too complex (in the sense that there are too many inputs and outputs).

The CIMOSA enterprise activity representation, which is compliant to the GAM model, proposes a simpler box with 6 legs (Figure 2). All inputs and outputs are semantically welldefined and have a formal definition in the model (Vernadat, 1993). Most of them are object views except the control output which provides the ending status of the activity. The control input and resource output must be information object views while the resource input must be a set of object views on enterprise objects playing the role of functional entities.

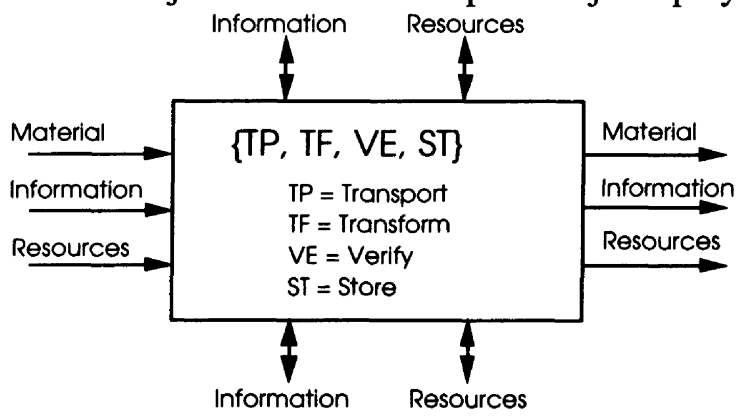

Figure 1 Generic Activity Model (GAM) representation

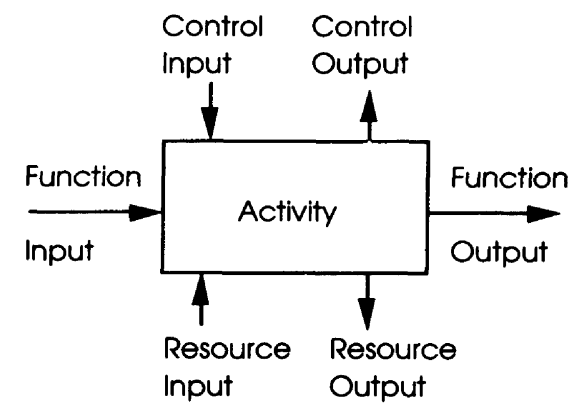

Figure 2 CIMOSA Enterprise Activity

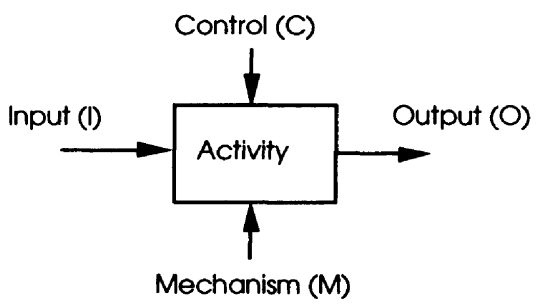

Figure 3 ICOM activity representation (IDEF0)

A simpler activity representation, based on actigrams of SADT and used in IDEF0 (ICAM, 1981), GIM (Vallespir et al., 1991) and IEM (Mertins et al., 1993) is often used in practice. It consists of a box with 4 legs, "called the ICOM (input, control, output, mechanism) activity box (Figure 3). It can be used to represent either processes and activities. 
Graphical notations are useful tools to communicate the results of a modelling case study but are not precise enough and sufficient to completely and formally model a complex system like a manufacturing enterprise. Modelling constructs in the form of templates or object classes and supporting languages are necessary. The next sections present essential features of activity and process constructs as required by enterprise modelling.

\subsection{Enterprise activity specification}

Enterprise activities are elementary functional units of an enterprise which require the allocation of time and resources for their execution. They define the enterprise functionality (i.e. the things to be done). Since all real-world objects can only be handled by their object views or as data, most inputs and ouputs of an enterprise activity are therefore defined as object views or sets of object views or even streams of object views (i.e. unbounded flows of object views for continuous activities).

Any activity is therefore characterised by:

- its name (or identifier),

- its set of objectives, constraints, declarative rules and description,

- its function input (FI), i.e. the set of or stream of object views to be transformed (information or physical object views),

- its function output (FO), i.e. the set of or stream of object views transformed by the activity (information or physical object views),

- its control input (CI), i.e. the set of object views used as control or constraints but not transformed by the activity (information object views only),

- its control output (CO), i.e. the ending status returned at the end of the activity execution and defined over a finite set of 0 -argument predicates providing the list of all the possible termination statuses of the activity,

- its resource input (RI), i.e. the set of object views on enterprise objects used as resources (functional entities) required to execute the activity,

- its resource output (RO), i.e. information object view(s) on enterprise object(s) used as resource input reporting on resource utilisation (optional),

- ts minimum and maximum durations (real numbers $d \min$ and $d \max$, dmin $<=d \max$ ), defining the time it takes to execute the activity ( $\mathrm{dmin}=\mathrm{dmax}$ for deterministic activities and $d_{\text {min }}$ _dmax for stochastic activities). An average duration with standard deviation could also be specified,

- its activity behaviour specifying its transformation function $f$ (algorithm or script indicating how its functional operations are employed),

- its capability set of required capabilities,

- its exact list of ending statuses.

Triggering conditions, i.e. conditions to be met to be able to execute the activity, must also be specified. They may depend on a combination of causal relations (e.g. which activities have just finished with which ending statuses), system states (defined by states of object views) and synchronisation conditions (e.g. clock times and time delays). They are defined and used as part of process behaviour in the next section. 
The transformation function $\mathrm{f}$ defines the activity behaviour, i.e. the set and ordering of elementary actions performed by the activity. These elementary actions are called functional operations. These are atomic operations performed on request by active resources or agents of the system. These active resources are called functional entities (AMICE, 1993; Vernadat, 1993). Each functional operation is formally denoted as (by analogy to a message sent to a method of an agent):

\section{FE.FU (parameter-list)}

where FE is the functional entity able to execute the functional operation FU and parameterlist is the list of (input/output) arguments of the operation.

The activity behaviour implementing the transformation function of an activity can generically specified as in CIMOSA by (Vernadat, 1994):

Activity Behaviour : \{ <A-behaviour $>$ [Exception Handling : <exceptions $>$ ] Ending Statuses <ending-statuses $>$

$<$ A-behaviour $>:=<$ declarations $><$ pre-conditions $><$ statements $><$ post-conditions $>$

where $<$ declarations $>$ is used to declare local variables, $<$ pre-conditions $>$ is a set of predicates defining pre-conditions on the execution of the activity, <statements> are Pascal-like procedural statements (including assignments, if-then structures, case structures, loops, etc.) defined on functional operations, <post-conditions > defines a (possibly empty) set of postconditions to be verified/set at the end of the activity and <ending-statuses> is the list of 0argument predicates defining the set of ending statuses of the activity. The <exceptions> clause (optional) allows the definition of exception handling mechanisms (such as time-outs or watch-dogs) to face non-deterministic situations (e.g. detection of an infinite loop, conditions never realised, deadlock situations, etc.). In this case, the control is passed either to an exception handling process or to a supervisor.

\subsection{Enterprise activity classification}

Different classes of activities exist in an enterprise and require specific definitions. We have:

- Machine Activities: Any machine activity A, i.e. activity performed by some device(s) without direct human intervention, is such that:

$-\mathrm{FI}_{\mathrm{A}} \cup \mathrm{FO}_{\mathrm{A}} \cup \mathrm{CI}_{\mathrm{A}} \_$$\varnothing ; \mathrm{FI}_{\mathrm{A}} \cap \mathrm{CI}_{\mathrm{A}}=\varnothing$;

$-\mathrm{FI}_{\mathrm{A}}, \mathrm{FO}_{\mathrm{A}}, \mathrm{CI}_{\mathrm{A}}, \mathrm{RO}_{\mathrm{A}} \subseteq V_{\mathrm{S}} ; \mathrm{RI}_{\mathrm{A}} \subseteq R \mathrm{~S}$;

- $\mathrm{CO}_{\mathrm{A}}$ is defined over a finite set of 0 -argument predicates;

- $\mathrm{f}_{\mathrm{A}}$ is a deterministic algorithm or an inference engine such that: $\left(\mathrm{FO}_{\mathrm{A}}, \mathrm{CO}_{\mathrm{A}}, \mathrm{RO}_{\mathrm{A}}\right)=$ $\mathrm{f}_{\mathrm{A}}\left(\mathrm{FI}_{\mathrm{A}}, \mathrm{CI}_{\mathrm{A}}, \mathrm{RI}_{\mathrm{A}}\right)$

where $\varnothing$ is the empty set, $V_{S}$ is the set of all object views of system $S, R S$ is the set of physical object views on objects used as resources in system $S$ and CIA $\cup R_{A} \subseteq I V S, I V S$ $\subseteq V_{S}$ is the set of information object views of system S. According to ISO (ISO, 1990), any machine activity can be classified as follows under the constraints reported in Table 1: 
- Make (e.g. transform, compute, decide, reason, ...)

- Move (e.g. load, unload, transport, return, ...)

- Verify (e.g. control, check, compare, ...)

- Rest (e.g. wait, in store, idle, ...)

Table 1

\begin{tabular}{|c|c|c|c|c|}
\hline FI & FO & $\mathrm{CI}$ & $\mathrm{CO}$ & RI \\
\hline Make & $\varnothing$ & $\varnothing$ & & $\varnothing$ \\
\hline Move & $\varnothing$ & FI & $\varnothing$ & $\varnothing$ \\
\hline Verify & $\varnothing$ & $\varnothing$ & $\varnothing$ & $\varnothing$ \\
\hline Rest_ $\varnothing$ & & & $\varnothing$ & $\varnothing$ \\
\hline
\end{tabular}

In these cases, the resource input can indicate the exact resource requirement, i.e. the functional entities to be used (early binding).or a list of alternative functional entities, i.e. the allocation of resources to activities is done at run-time (late binding).

- Human Activities: Human activities are activities performed by human beings, i.e. their resource inputs are human operators. Although the general formalism defined above still applies to human activities, the control input is in this case made of object views of the system or of less formal things such as decision variables, expert advices or knowledge about some technical domain. The transformation function is no more a deterministic algorithm and the time duration is of course nearly always stochastic because of human presence. Moreover, the definition of the set of ending statuses becomes much more difficult and its size may be large.

No widely accepted classification of human activities currently exists. Relevant human activities for CIM systems involve: knowledge acquisition, thinking/reasoning, creating, decision-making, executing, communicating, and control.

- Hybrid Activities: Hybrid activities are activities which cooperatively involve both machines and humans as their functional entities. The transformation function can be an algorithm or a script (defining some procedure to be followed by resources). However, interpretation of the script in terms of the sequence of functional operations to be performed can be left to the appreciation of the human operators involved.

- Cooperative Activities: Cooperative activities are activities which involve the exchange of messages, data, information or object views and need synchronisation. Thus, they make use of the following basic functional operations:

- request (to ask for message or data or object views)

- receive (message, data or object views)

- send/broadcast (message, data or object views)

- acknowledge (for synchronisation purpose) 


\section{BUSINESS PROCESSES}

Activities can be organised into sequences of actions to form processes. Processes can be organised into larger processes. Processes represent the flow of control in an enterprise (i.e. they represent the enterprise behaviour).

A business process is a partially ordered set of activities (or sub-processes). Processes are triggered by the occurrences of events and execute until they have produced their end-result (defined as object views) or are interrupted.

Events are solicited or unsolicited real-world happenings (e.g. machine break-downs, orders, triggers, clock times, system changes). Events can be either internal (endogeneous) or external (exogeneous) to a system. An event happens at a given time and may carry information in the form of an information object view.

Two kinds of business processes can be defined (Bussler, 1994):

- structured processes, for which the sequence of activities is completely defined; and

- ill-structured processes, for which the sequence of activities is partially defined or unknown.

In computer theory (Curtis et al., 1992; Hoare, 1985; Milner, 1980), it has been shown that activites can be combined into processes using only one operator, the parallel operator denoted "II", to describe any flow of control assuming that this operator is not commutative (Milner, 1980). A more explicit definition of processes is preferred for enterprise modelling using control structures such as sequence, parallelism, rendez-vous, choice or loop to link activities in a causal network (AMICE, 1993; Vernadat, 1993). These control structures are all defined as WHEN <condition> DO <action> rules (called behavioural rules). These rules make use of the ending status function ES previously defined on enterprise activities.

A business process is characterised by:

- its name,

- its set of triggering events,

- its process behaviour defined as a set of behavioural rules (of the form WHEN $<$ conditions $>$ DO <actions $>$ ),

- its set of ending statuses (defined as a logical combination of ending statuses of the employed activities).

Any kind of process behaviour can be specified as (where $<\mathrm{P}$-behaviour $>$ is a list of behavioural rules):

Process Behaviour : $<\mathrm{P}$-behaviour $>$ End Process

$<\mathrm{P}$-behaviour $>::=<\mathrm{P}$-statement $><$ next-P-statement $>$

$<$ next-P-statement $>::=<$ P-statement $><$ next-P-statement $>$ I NIL

$<$ P-statement $>::=$ WHEN $(<$ B-conditions $>)$ DO $<$ actions $>$

\subsection{Structured processes}


Their process behaviour is deterministically defined by the following types of rules (in which EF stands for enterprise function and can represent either an enterprise activity or a business process):

- Process triggering rules: They are used to start a process by means of events.

WHEN (START WITH event-i) DO EF1

The process starts with function EF1 any time that an occurrence of event-i occurs.

- Forced sequential rules: They are used when a function EF2 must follow another function EF1 whatever the ending status of EF1 is. The reserved word 'any' is used in this case (not an ending status).

WHEN $(\mathrm{ES}(\mathrm{EF} 1)=$ any $) \mathrm{DO}$ EF2

- Conditional sequential rules: They are used to represent branching conditions in the flow of control.

WHEN $(E S(E F 1)=$ end_stat_1) DO EF2

WHEN $(E S(E F 1)=$ end_stat_2) DO EF3

WHEN (ES(EF1) = end_stat_3) DO EF4

- Spawning rules: They are used to represent the parallel execution of enterprise functions in a flow of control. Two types of spawning rules can be defined:

(a) Asynchronous spawning: When EF1 is finished with status 'value', EF2, EF3 and EF4 are requested to start as soon as they are ready ( $\&$ is the parallel operator).

WHEN $(\mathrm{ES}(\mathrm{EF} 1)=$ value $)$ DO EF2 \& EF3 \& EF4

(b) Synchronous spawning: When EF1 is finished with status 'value', EF2, EF3 and EF4 are all requested to start exactly at the same time (SYNC indicates the synchronisation).

WHEN $(E S(E F 1)=$ value) DO SYNC (EF2 \& EF3 \& EF4)

- Rendez-vous rules: They are used to synchronise the end of spawning rules.

WHEN $(E S(E F 2)=$ value_2 $A N D$ ES(EF3) = value_3 AND ES(EF4) = value_4)

DO EF5

- Loop rules: They are used to execute again the same type of enterprise function.

WHEN (ES(EF1) = loop_value) DO EF1

- Process termination rules: They are used to indicate the end of the process.

WHEN $(E S(E F 2)=$ end_stat_x AND ES(EF2) $=$ end_stat_y) DO FINISH

Using these rules, a process behaviour is said to be consistent if FINISH can be reached from all STARTs and all enterprise functions used in the rules belong to at least one path from START to FINISH.

\subsection{Ill-structured processes}

Ill-structured processes are processes for which the exact sequence of all employed activities and/or sub-processes is not completely known. In this case, temporal logic (Zaytoon et al., 1993) can be used and the process behaviour becomes:

Process Behaviour: <behavioural rules $>$ [Subject To $<$ temporal rules $>$ ]

End Process

Two types of behavioural rules are added to the previous set to model ill-structured processes: random choice rules and open set rules. In these rules, the action part is reinterpreted 
(variable S), meaning that it is considered as a whole to make possible the definition of its ending status.

- Random choice rules: These rules are used when there is an exclusive choice among several alternatives. Exactly one function in the list will be executed as decided by the resource at run-time, which must be common to all functions in the list.

WHEN $(E S(E F 1)=$ end_stat_1) DO S $=($ EF2 $\mid$ EF3 $\mid$ EF4 $)$

- Open set rules: These rules say that a set of functions must be executed next but the order is unknown. In this case, all functions are supposed to be executed.

WHEN $(E S(E F 1)=$ end_stat_1) DO S $=\{E F 2, E F 3, E F 4\}$

The flow of control for the open set of functions of the open set rules will be governed by temporal rules defined as follows:

- A Before B: to specify that A must be executed sometime before B

- A Meets B: to specify that B starts just after A ends

- A During B: to specify that A is executed after B starts but during B

- A Starts B: to specify that when A starts, B must start

- A Finishes B: to specify that when A ends, B must end

The complete flow of activity execution of a process is only known upon completion of the process (as well as its ending status). This flow of activities over time is called the trace of the process.

\section{EXAMPLES}

Let us consider the process depicted by Figure 4.

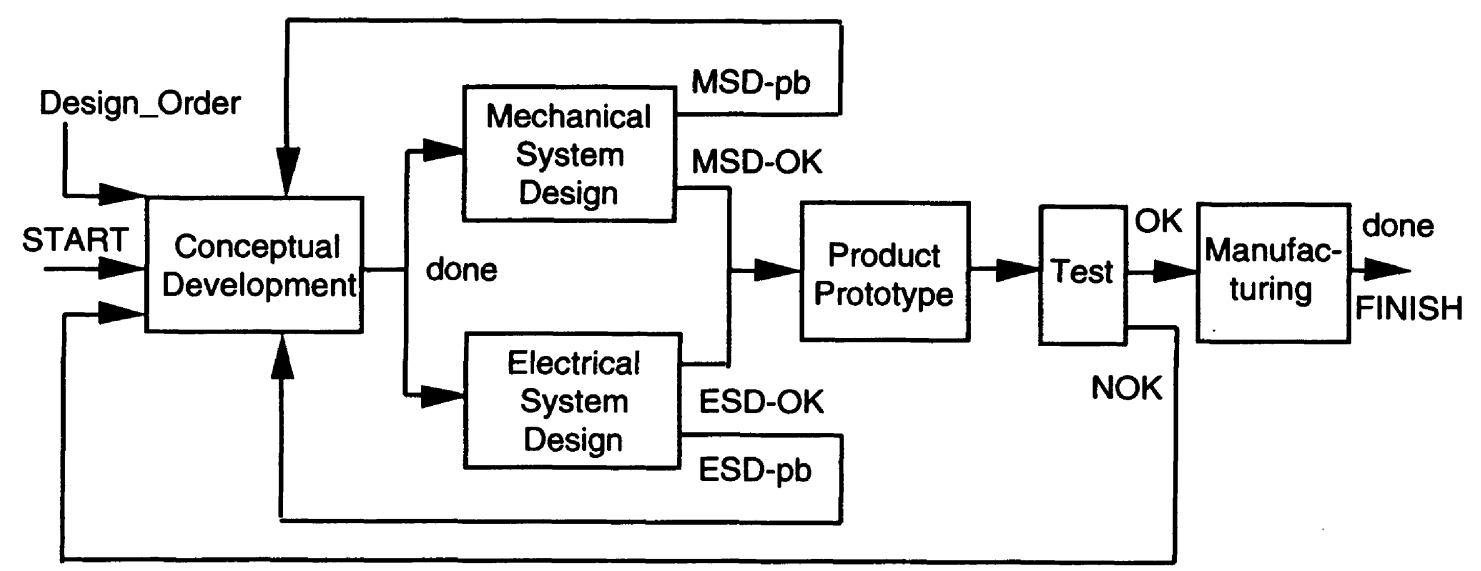

Figure 4 A product design process

It describes an engineering process for designing and testing a product made of a mechanical system and an electrical system. The process starts with a conceptual development activity. 
When it is done the process continues with two parallel sub-processes, one for the mechanical system design and one for the electrical system design. If both sub-processes finish successfully (ending statuses MSD-OK and ESD-OK respectively), the process goes for product prototype and then for test. If the test is not successful (ending status NOK), the process goes back to conceptual development. This also happens if either mechanical system design or electrical system design encounters problems (ending statuses MSD-pb and ESD-pb respectively). If the test is successful then the process continues for manufacturing and finally ends. The process is specified as follows:

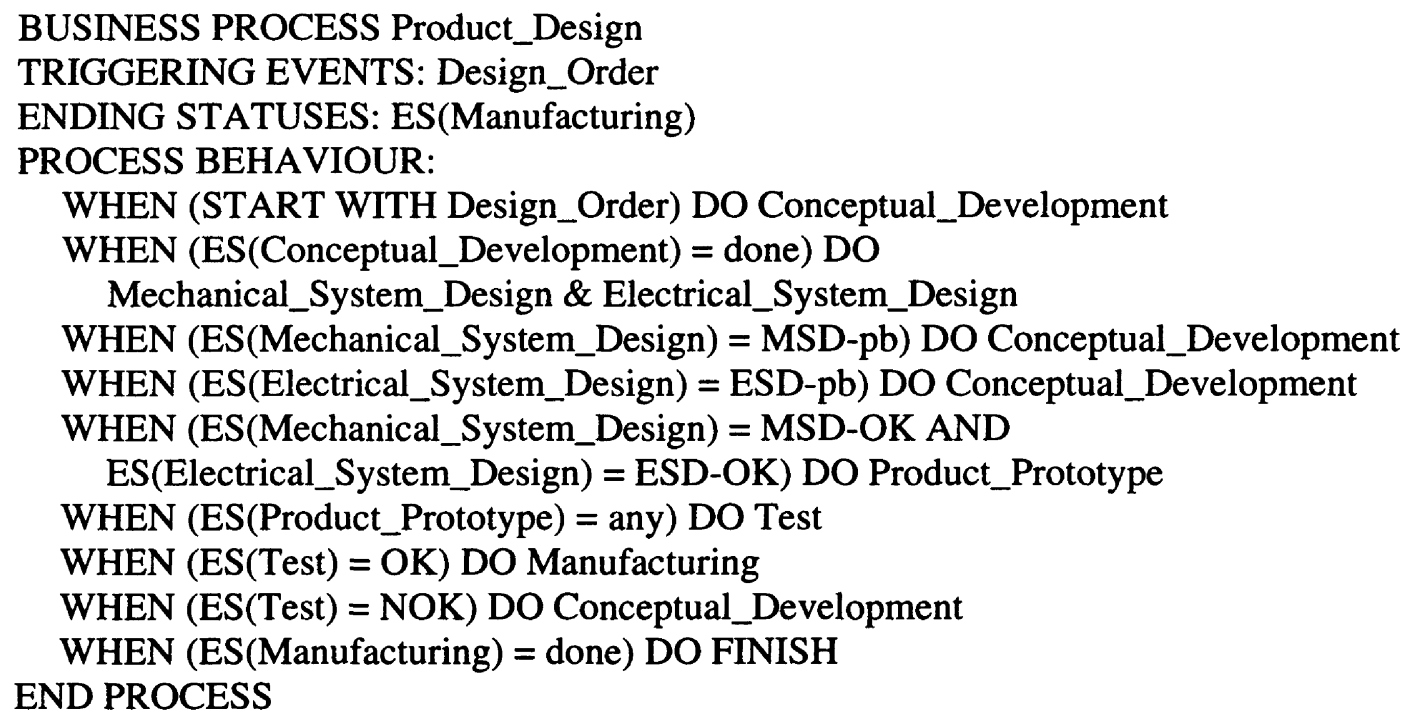

Let us now assume that the sub-process Electrical_System_Design is a ill-structured process made of three basic activities: Rotor_Design, Stator_Design, Body_Design. Some designers may prefer to start with Rotor_Design, others with Stator_Design. We can specify the Electrical_System_Design process as follows:

BUSINESS PROCESS: Electrical_System_Design

TRIGGERING EVENTS:

ENDING STATUSES: ESD-OK, ESD-pb

PROCESS BEHAVIOUR:

WHEN (START) DO A $=$ \{Rotor_Design, Stator_Design, Body_Design $\}$

WHEN $(E S(A)=$ completed $)$ DO FINISH

SUBJECT TO

Rotor_Design BEFORE Body_Design;

Stator_Design BEFORE Body_Design

END PROCESS

In this example, ES $(\mathrm{A})=\mathrm{ES}($ Body_Design $)=$ completed. 


\section{CONCLUSION}

In this paper, a formalism to represent business processes and enterprise activities has been defined. It is based on the principle of separation of enterprise behaviour and enterprise functionality as promoted by CIMOSA and considered as a fundamental modelling and organisation principle. Adherence to this principle provides organisational flexibility in the model because enterprise behaviour can be easily updated without affecting enterprise functionality and vice versa. This is an essential property for easy management of change as required by continuous process improvement (CPI) for improving enterprise responsiveness.

Enterprise behaviour is modelled by means of a declarative language based on WHEN-DO condition rules while enterprise functionality is modelled as a chunk of elementary functionality and can be implemented in terms of a procedural language defining the procedure of execution of the functional operations of the transformation of the activity.

\section{REFERENCES}

AMICE (1993) CIMOSA: Open System Architecture for CIM, 2nd revised and extended version. Springer-Verlag, Berlin.

Bussler, C. (1994) Enterprise process modeling and enactment in GERAM, Proc. Third Int. Conf. on Automation, Robotics and Computer Vision (ICARCV'94), Singapore

CEN (1990). CEN/CENELEC. ENV 40 003: Computer-Integrated Manufacturing. Systems Architecture. Framework for Enterprise Modelling. CEN, Brussels.

Curtis, B., Kellner, M.I., Over, J. (1992) Process modeling, Comm. of the ACM, 35(9), 75-90.

Hoare, C.A.R. (1985) Communicating Sequential Processes. Prentice-Hall, Englewood Cliffs, NJ.

ICAM (1981). Integrated Computer-Aided Manufacturing (ICAM) Architecture - Part II, Vol. IV (IDEF0), Vol. V (IDEF1), Vol. VI (IDEF2), SofTech Inc, Waltham, MA.

ISO (1990) Reference Model for Shop Floor Production Standards. Technical Report 10314, Part 1. ISO TC 184/SC5/WG1 N126) and Part 2 (ISO TC 184/SC5/WG1 N160).

Mayer, R.J., Cullinane, T.P., deWitte, P.S., Knappenberger, W.B., Perakath, B. and Wells, M.S.(1992) Information Integration for Concurrent Engineering (IICE) IDEF3 Process Description Capture Method Report. Armstrong Laboratory, Wright-Patterson AFB, Ohio 45433, AL-TR-1992-0057.

Mertins, K., Süssenguth, W., Jochem, R. (1993) Planning of enterprise-related CIM structures. In Advances in Factories of the Future, CIM and Robotics (ed. M. Cotsaftis and F. Vernadat), Elsevier, Amsterdam. pp. 67-76.

Milner, R. (1980) A Calculus of Communicating Systems. Lecture Notes in Computer Science 92, Springer Verlag, New York.

Rumbaugh, J. et al. (1991) Object-Oriented Modelling and Design. Prentice-Hall, Englewood Cliffs, NJ.

Vallespir, B., Chen, D., Zanettin, M., Doumeingts, G. (1991) Definition of a CIM architecture within the ESPRIT Project IMPACS. In Computer Applications in Production Engineering: Integration Aspects, Elsevier, Amsterdam. pp. 731-738. 
Vernadat, F. (1993) CIMOSA: Enterprise modelling and enterprise integration using a process-based approach. In Information Infrastructure Systems for Manufacturing, North-Holland, Amsterdam (ed. H. Yoshikawa and J. Goossenaerts). pp. 65-84.

Vernadat, F. (1994) A language for CIM system specification, Proc. ISPE/IFAC 10th Int. Conf. on CAD/CAM, Robotics and Factories of the Future, Ottawa, Canada. pp. 283288.

Vernadat, F (1995). Enterprise Modeling and Integration Principles, Chapman \& Hall, London, (in press).

Zaytoon, J., Niel, E., Mille, A. and Jutard, A. (1993) A temporal SADT for automated manufacturing systems, Proc. Int. Conf. on Industrial Engineering and Production Management (IEPM'93), Vol. I, Mons, Belgium. pp. 154-164.

\section{BIBLIOGRAPHY}

Dr. François Vernadat is a French and Canadian citizen. He got his Ph.D. degree in Electrical Engineering in 1981 from University of Clermont, France. Since then, he has held research officer positions at the National Research Council of Canada (NRCC) from 1981 till 1988 and INRIA, a French research center for computer science and automatic control, from 1988 till now. He has authored or co-authored over 100 technical papers on syntactic pattern recognition, CIM databases and information system design methodologies $\left(\mathbf{M}^{*}\right)$, enterprise modelling, enterprise integration, Petri nets and manufacturing plant layout. He has participated in several ESPRIT projects and was one of the chief architects of CIMOSA.

$\mathrm{He}$ is a co-editor of several edited books on CAD/CAM, CIM and Factories of the Future and an author of the books Practice of Petri Nets in Manufacturing and Enterprise Modeling and Integration Principles, both published by Chapman and Hall, London. He is currently the European editor of the International Journal of Computer-Integrated Manufacturing. He has been co-chairman of several international conferences on industrial engineering and automation and chairman of the European Workshop on Integrated Manufacturing Systems Engineering held in 1994 in Grenoble, France. He is a member of ACM, IEEE and SME. 